\title{
Nonlinearity in Complexity Science
}

\author{
R.S.MacKay \\ Mathematics Institute and Centre for Complexity Science \\ University of Warwick, Coventry CV4 7AL, U.K.
}

August 25, 2008

\begin{abstract}
The role of nonlinearity in complexity science is discussed, and some nonlinear research problems in Complexity Science are sketched in the contexts of the buzzwords "emergence, interacting agents and swarms".
\end{abstract}

Keywords: nonlinearity, complexity science, emergence, interacting agents, swarms

\section{Introduction}

Complexity Science is experiencing a new vogue. The doubts of the previous decade (e.g. [Ho]) seem to have lost their force. Stephen Hawking declared that the 21st century "will be the century of complexity" [Ha]. Complexity Science is receiving serious funding from the European Commission, UK Engineering and Physical Sciences Research Council, and other agencies.

There are many interpretations of the term "complexity science", e.g. [Man]. I use it to mean the study of systems with many interdependent components, cf. [BY]. I think most "complexity scientists" would accept this definition, though some would wish to add words like "adaptive" or "emergent phenomena".

How does "nonlinearity" enter Complexity Science? For some (mainly philosophers and social scientists), "nonlinearity" means that the causal links of the system form something more complicated than a single chain, for example a system with feedback loops. A complex system is then one with an intricate graph of causal links, thus highly nonlinear in this sense.

For others, however (including I think the founding editors and most authors and readers of this journal), "nonlinearity" means that the superposition principle does not apply. A system is linear if one can add any two solutions and obtain another, and multiply any solution by any factor and obtain another. Otherwise, it is nonlinear.

According to many, the core of Complexity Science is Nonlinear Dynamics (with nonlinearity understood in the sense of failure of the superposition principle), e.g. Fritzhof Capra in his plenary lecture at the Complexity, Science and Society conference in Liverpool (Sept 2005), and several textbooks (e.g. [BY, BV, Er]). This view requires some 
caveats, however, so I don't regard it is the primary role of nonlinearity in complexity science. Firstly, there are many complexity science problems that are about statics, e.g. complex structures, complex materials, equilibrium statistical mechanics and percolation. Secondly, my definition of complexity science excludes low-dimensional dynamical systems (unless one thinks of the states at successive moments of time as many interdependent components) and therefore low-dimensional chaos, a central topic in nonlinear dynamics. Thirdly, there are dynamical complex systems which would not normally be covered by the term "nonlinear dynamics": stochastic, game-theoretic and quantum dynamics; what constitutes nonlinearity needs careful formulation for these. Fourthly, there are linear complexity science problems, e.g. random matrix theory and the linear response of a network.

I consider stochastic models as taking centre stage in complexity science. At the level of probability distributions, however, stochastic models are linear: any convex combination of allowed probability distributions for a problem is also allowed (and that makes the problem essentially linear). Thus it might seem that there is no nonlinearity for this important class of complex systems. Yet there is a sense in which a superposition principle may still fail. It is easiest to see for systems that conserve some quantity, let's call it number of particles. Then I propose to call the system linear if one can "superpose" solutions for $n_{1}$ and $n_{2}$ particles and obtain a solution for $n_{1}+n_{2}$ particles, and nonlinear if this is not always true. Here, superposition means that the joint probability distribution is a product, that is the particles behave independently. Thus in this view, nonlinearity corresponds precisely to the word "interdependent" of the definition of complexity science.

A similar view of nonlinearity can be formulated for quantum systems conserving particle number or amount of action (cf. [M02], where I focussed on "anharmonicity" as an expression of the latter). Similarly, game-theoretic models can be considered nonlinear if one doesn't always obtain solutions for $N$ players by superposition (as is the case in all non-trivial models). Again nonlinearity in this sense corresponds to dependence (between the players).

Thus I propose that "nonlinearity" in complexity science means "dependence".

Sometimes, some or all dependence can be removed by a relatively simple change of view. For example, a linear (in the standard sense) dynamical network can be analysed into normal modes which evolve independently; if the eigenvector problem is relatively straightforward, I'd say this system is not complex.

It is less clear how to define "nonlinearity" for quantum or stochastic systems with no conserved quantity, but I suggest that the basic criterion should be that there is no simple change of view to independent entities. Thus if there is no concept of conserved entity then a fortiori the system is nonlinear.

On the basis that nonlinearity means dependence, I present here a personal selection of nonlinear mathematical problems in Complexity Science, around some of the key buzzwords: emergence, interacting agents, and swarms, concluding with a miscellany. Space and time limit me from listing all the relevant references, so I apologise for the incompleteness. 


\section{Emergence}

One of the most seductive buzzwords of complexity science is "emergence". Arguments rage about what it means. To me, emergence means non-unique statistical behaviour without any topological reason. I came to this point of view when my $\mathrm{PhD}$ student David Sanders asked in October 2000 for a project on emergence.

Note that emergence is very different from chaos, in which sensitive dependence produces highly non-unique trajectories according to their initial conditions. Indeed, the nicest forms of chaos produce unique statistical behaviour in the basin of an attractor. The distinction is like that between the weather and the climate. For weather we care about individual realisations; for climate we care about statistical averages.

A way to make the concept of statistical behaviour mathematically precise is the notion of "space-time Gibbs phase". This is most easily introduced in the context of probabilistic cellular automata (PCA). A PCA is a discrete-time Markov process on a product space $X=\prod_{s \in S} X_{s}$, where $S$ is a countable metric space (often $\mathbb{Z}$ or $\mathbb{Z}^{2}$ in examples, but there is no requirement for $S$ to be translation invariant nor even infinite), each $X_{s}$ is a finite set, the states $x_{s}^{\prime} \in X_{s}$ at the next time are conditionally independent for different $s \in S$ given the current state $x=\left(x_{r}\right)_{r \in S}$, and $x_{s}^{\prime}$ is independent of $\left(x_{r}\right)_{r \in S \backslash N(s)}$ for some bounded neighbourhood $N(s)$ of $s \in S$. Thus it can be specified by the transition probabilities $p\left(x_{s}^{\prime} \mid\left(x_{r}\right)_{r \in N(s)}\right)$.

The space-time Gibbs phases for a PCA are the probability distributions one can expect to see for the state of arbitrary bounded pieces of space-time $S \times \mathbb{Z}$, given that the process was started in the infinite past. They are determined by the self-consistency conditions that for any bounded $\Lambda \subset S \times \mathbb{Z}$, the probability distribution of the state on $\Lambda$ given the state on the domain $B \subset S \times \mathbb{Z}$ influencing $\Lambda$ (excluding $\Lambda$ itself) is given by multiplying the transition probabilities to make up the state on $\Lambda$ from that on $B$. It is convenient to write the product as $\exp \left(-\sum_{s t \in \Lambda} \phi_{s t}(x)\right)$ where $\phi_{s t}(x)=-\log p\left(x_{s}^{t} \mid x_{N_{s}}^{t-1}\right)$, which makes it resemble the concept of Gibbs phase in equilibrium statistical mechanics.

For finite $S$ then $X$ is finite, so if the Markov chain is irreducible (or more generally if it has a unique communicating component) then there is a unique space-time Gibbs phase and it is the one generated by iterating the unique stationary probability measure on $X$ for the chain.

Just as in equilibrium statistical mechanics, however, the idealisation of spatially infinite systems is useful, and then there can be more than one space-time Gibbs phase even if the process is irreducible. A nice example is Toom's NEC majority voter model $[\mathrm{To}+]$, in which the underlying network $S$ is $\mathbb{Z}^{2}$, the local state spaces $X_{s}$ are $\{+,-\}$ and at each time step the state at each site is updated to the majority state of its north-eastcentre neighbourhood with probability $1-\varepsilon$. For $\varepsilon$ small enough, it exhibits at least two space-time Gibbs phases, corresponding to two stationary probability measures. The set of space-time Gibbs phases is always convex, so when we count them we really refer to extremal ones (those which are not convex combinations of others). Even more interesting, moving $\varepsilon$ near to 1 produces an "anti-majority voter" model which again exhibits at least two phases, neither of which corresponds to a stationary measure [GM]. 
Instead they correspond to two "phases", in the sense of phase of an oscillator, of a space-time probability distribution with period two in time. Thus non-trivial collective behaviour "emerges".

A catch with this view of emergence is that it applies only to spatially infinite systems. But just as in equilibrium statistical mechanics, it has finite system analogues. The basic one is that non-unique phase corresponds to long-range correlations. A quantitative measure of emergence has been proposed in terms of mutual information [BlM].

The framework of space-time Gibbs phases allows one to develop general questions, e.g.

Problem: Determine classes of system for which the set of space-time Gibbs phases moves continuously with parameters. A start has been made in [M07a] for some cases where this set is a single point; it is shown to move smoothly with respect to parameters with respect to a suitable metric on the space of probability measures on a product space.

Problem: Understand how to steer the set of space-time Gibbs phases for a system towards a desired one, e.g. to make artificial models of small-scale weather for use in climate prediction.

Problem: Develop bifurcation theory for space-time Gibbs phases.

Problem: Understand the effects of controls on space-time Gibbs phases; determine conditions under which local control has only local effect or can have long-range effect; consider the effects of boundaries (open systems).

Problem: Develop renormalisation theory for space-time Gibbs phases, and the resulting concept of universality class. Despite its having become a standard part of theoretical physics, it is not clear to me that this is really worked out even in equilibrium statistical mechanics (but see [Br], and [M95] for an example and [CM] for its quantum version), or percolation.

Problem: Devise methods to fit space-time Gibbs phases to data, cf. [CFL]. A substantial issue is the lack of uniqueness in the Gibbs potential, so one should develop a concept of a simplest potential modulo co-boundaries.

The concept of space-time Gibbs phase can be extended to some classes of deterministic spatially extended dynamical system. The simplest such class is expanding coupled map lattices $f: X \rightarrow X$ with a Markov partition. Then the space-time solutions correspond (up to ambiguities at the boundaries of partition elements) to space-time symbol tables with finite symbol set $\Sigma_{s}$ for each site and the space-time Gibbs phases are given by the consistency condition for $\phi_{s t}(\sigma)=\operatorname{tr}\left(\log D f\left(x^{t}(\sigma)\right)\right)_{s s}, \sigma \in\left(\prod_{s \in S} \Sigma_{s}\right)^{\mathbb{Z}}[\mathrm{BrKu}](\mathrm{I}$ proposed this formula independently in Feb 95; see remark in [M96]). This allowed us to make a coupled map lattice exhibiting the same results as Toom's voter PCA [GM]. The Gibbs potential generalises to invertible CML with Markov partition by restricting $D f$ to its backward contracting subspace. A way to make our CML example invertible was given in [M05] but it is not uniformly hyperbolic (nor even continuous), so lacks the robustness (structural stability) that uniform hyperbolicity would confer. The example of $[\mathrm{GM}]$ was modified to a continuous (even $C^{\infty}$ ) example in $[\mathrm{BaKe}]$ but the effect of their coupling is not surjective, which raises the following 
Problem: Make a smooth invertible spatially extended indecomposable deterministic dynamical system exhibiting non-unique space-time Gibbs phase.

The above examples have spatially asymmetric coupling, because they are based on Toom's NEC voter PCA, but many would prefer a spatially symmetric example, so

Problem: Solve the previous problem with spatially symmetric coupling (possibly by making a spatially symmetric version of Toom's PCA).

It would also be good to extend to continuous-time deterministic systems, e.g.

Problem: Make arbitrarily high dimensional mechanical systems with Anosov dynamics on each energy level. I think this could be achieved by a generalisation of the triple linkage $[\mathrm{HM}]$ to an array of rotating disks coupled by rods. Even more interesting would be to make a spatially infinite array with non-unique space-time Gibbs phase!

There are many interesting phenomena for deterministic spatially extended dynamical systems which to me should be interpreted as space-time Gibbs phases, e.g.

Problem: Explain collective oscillations in large Hamiltonian systems, such as [MK] (and earlier references).

Problem: Explain stable chaos [PLOK].

Here are some final questions for this section:

Problem: Derive rigorous results for self-organised criticality (for background, see [Je]).

Problem: Develop the concept of hierarchical emergence, i.e. a sequence of levels of emergence, cf. [An].

There are complex systems which perhaps never settle down, e.g. biological evolution or gravitational systems, or where the interest is in the long-time relaxation, e.g. aggregation and coarsening models. For such systems space-time Gibbs phases are not such a useful concept.

Problem: Find some models for which you can prove there are no space-time Gibbs phases.

\section{Interacting agents}

This buzzword is central to Complexity Science. I tend to restrict its use to cases where an agent's behaviour is the result of optimisation for himself, at least approximately, based on limited knowledge of his surroundings and the past. Even for a single agent with deterministic environment, results can be quite intricate, e.g. [MSS].

The behaviour of interacting agents can involve "learning" or "adaptation". The minority game [Coo] is an example. Some people regard adaptation as an essential feature for a complex system, but I regard it simply as adding a longer time-scale component to the dynamics, so not a fundamental change.

The concept of an agent as a maximiser of its utility function (a function of their own and other agents' actions) is central to economics. I think, however, it is too idealised (probably most economists would agree). One way to relax the ideal is to suppose that agents make imperfect optimisation according to some probabilistic rule. For example, one can make imperfect optimisation according to a logit (Glauber) or Metropolis choice, which then reduces a many-agent dynamic game to a PCA (or similar in continuous time) 
and hence to the context of section 2. With this view, a utility function reduces to the exponential of a transition probability (up to normalisation).

Problem: Develop space-time Gibbs phases for such interacting agents.

A fundamental question for interacting agent systems is whether their selfish optimisation produces a socially desirable result. Fans of the free market claim the answer is yes, but there are many examples that show this is false, cf. [Ro]. To address the question thoroughly, one has to decide what constitutes a desirable result. One point of view is an optimum of some collective aggregate. Another is a phase from which any change reduces the utility for at least one agent (Pareto optimum). But neither is guaranteed by selfish optimisation.

Problem: Study how far space-time Gibbs phases might be from a social optimum or even from a Pareto optimum.

Another way in which to relax the economist's ideal is to endow an agent with only a partial order on his actions, rather than a utility function. For example, he may simultaneously wish to maximise wealth and being liked by others: if he can make an improvement in either without reduction in the other then he'll do so. In general this leads to non-unique outcomes. It also leads to inertia if there is an inhibition to making large changes.

Problem: Study the effects of partial-order agents.

The big challenge under this topic is to find "Newton's laws of society". This dream was born not long after Newton's laws of mechanics (see [Ba]) but remains open.

Problem: Find out how humans make decisions, and analyse the consequences for society.

\section{Swarms}

Much of the mathematical work on complex systems is for systems on a prescribed network, but there are many complex systems which do not fit this set-up. The links in the network may themselves evolve, or coupling may be via proximity in space. A prime example is swarms, e.g. [BDT]. The agents move through space and their interactions are principally with those which are nearby in space. A similar class of examples is spread of infections between mobile agents.

Problem: Develop the theory of space-time Gibbs phases for systems without a fixed network.

Problem: Prove existence of a liquid crystal phase for Sumpter's model of locusts [Su]; this is likely to be hard, because as far as I am aware there is not even a proved model of a liquid crystal phase in equilibrium statistical mechanics.

Problem: Study contact processes for swarms, e.g. spread of infections between mobile agents. 


\section{Miscellaneous}

Problem: Quantify the complexity of a multivariate probability distribution; a product distribution should have zero complexity. This could develop from the notion of "statistical complexity" for time series [FC].

Problem: Understand conditions for synchronisation in networks of dissimilar units via renormalisation and percolation; more generally mode-locking [M94]. Apply to the generators of breathing rhythm [FDN], and the millenium bridge [S+] (but use more general models of walkers, as I proposed to a student back in 2001).

Problem: Find conditions for robustness of network dynamics, e.g. build on [M96, MS, BM97, BiM].

Problem: Extend results on coherent structures, e.g. discrete breathers [M00a] and their movement/interaction [M04] (but construction of higher order slow manifolds is more complicated than suggested there [BM08]); break away from 1D spatial dynamics approach, e.g. [JM].

Problem: Develop general results on model reduction, e.g. derive Langevin equations for slow degrees of freedom of Hamiltonian systems, as sketched in [M07b], and develop applications e.g. [MM].

Problem: Develop models of nonlinear conductivity by nucleation of kink/antikink pairs and their opposite propagation, building on [BM04].

Problem: Study complex statics problems, e.g. complex materials, configurations of alloys (cf. [M00b]), radiation damaged steel.

Problem: Prove persistence of spectral projections for many-body quantum problems as sketched in [M00a], but cf. Yarotsky [Ya].

Problem: Understand nonlinear Anderson localisation, in both classical and quantum contexts.

Problem: Study complex systems from an information-processing view; for example, gene expression, neuronal networks.

Problem: Explain the arrow of time as a result of non-unique phase for some model of quantum gravity (probably need to link to matter/anti-matter balance too).

\section{References}

[An] Anderson PW, More is different, Science 177 (1972) 393-6.

[Ba] Ball P, Critical mass (Heinemann, 2004).

[BlM] Ball RC, MacKay RS, in preparation

[BaKe] Bardet J-B, Keller G, Phase transitions in a piecewise expanding coupled map lattice with linear nearest neighbour coupling, Nonlinearity 19 (2006) 2193-2210 (plus erratum at http:// )

[BY] Bar-Yam Y, Dynamics of complex systems (Westview, 1997).

[BM97] Baesens C, MacKay RS, Exponential localisation of linear response in networks with exponentially decaying coupling, Nonlinearity 10 (1997) 931-940. 
[BM04] Baesens C, MacKay RS, A novel preserved partial order for cooperative networks of overdamped second order units, and application to tilted FrenkelKontorova chains, Nonlinearity 17 (2004) 567-80.

[BM08] Baesens C, MacKay RS, Hamiltonian slow manifolds of high order and with internal oscillation, in preparation

[BV] Bertuglia CS, Vaio F, Nonlinearity, Chaos and Complexity (Oxford, 2005).

[BiM] Bishnani Z, MacKay RS, Safety criteria for aperiodically forced systems, Dyn Sys 18 (2003) 107-29.

[BDT] Bonabeau E, Dorigo M, Theaulaz G, Swarm intelligence (Oxford, 1999).

[BrKu] Bricmont J, Kupiainen A, High temperature expansions and dynamical systems, Commun Math Phys 178 (1996) 703-32.

[Br] Brydges DC, Lectures on the renormalisation group, Park City (Am Math Soc, 2007).

[CM] Catarino NR, MacKay RS, Renormalization and quantum scaling of FrenkelKontorova models, J Stat Phys 121 (2005) 995-1014.

[CFL] Chazottes J-R, Floriani E, Lima R, Relative Entropy and Identification of Gibbs Measures in Dynamical Systems, J Stat Phys 90 (1998) 697-725.

[Coo] Coolen AAC, The mathematical theory of minority games (Oxford, 2005).

[Er] Erdi P, Complexity Explained (Springer, 2008).

[FC] Feldman D, Crutchfield JP, Measures of statistical complexity: why? Phys Lett A 238 (1998) 244-252.

[FDN] Feldman JL, Del Negro CA, Looking for inspiration: new perspectives on respiratory rhythm, Nature Rev Neuro 7 (2006) 232-241.

[GM] Gielis G, MacKay RS, Coupled map lattices with phase transition, Nonlinearity 13 (2000) 867-88.

[Ha] Hawking S, San Jose Mercury News (23 Jan 2000).

[Ho] Horgan J, From complexity to perplexity, Sci Am (June 1995) 74-9.

[HM] Hunt TJ, MacKay RS, Anosov parameter values for the triple linkage and a physical systems with a uniformly chaotic attractor, Nonlinearity 16 (2003) 1499-1510.

[JM] James G, MacKay RS, Amplitude equations for time-periodic solutions of infinite-dimensional reversible systems, with application to 2D Klein-Gordon lattices, in preparation

[Je] Jensen HJ, Self-organized criticality (Cambridge, 1998).

[M94] MacKay RS, Mode-locking and rotational chaos for networks of oscillators: a mathematical framework, J Nonlin Sci 4 (1994) 301-14. 
[M95] MacKay RS, The classical statistical mechanics of Frenkel-Kontorova models, J Stat Phys 80 (1995) 45-67.

[M96] MacKay RS, Dynamics of networks: features which persist from the uncoupled limit, in: Stochastic and spatial structures of dynamical systems, eds Strien SJ van, Verduyn Lunel SM (N Holland, 1996) 81-104.

[M00a] MacKay RS, Discrete breathers: classical and quantum, Physica A 288 (2000) 174-98.

[M00b] MacKay RS, Defects in solids, large molecules and space-structures, Proc Roy Soc Lond A 456 (2000) 1883-95.

[M02] MacKay RS, Many-body quantum mechanics, in: Nonlinear dynamics and chaos, where do we go from here? eds Hogan J, Champneys A, Krauskopf B, Bernardo M di, Homer M, Wilson E, Osinga H (Inst of Phys, 2002) 21-54.

[M04] MacKay RS, Slow manifolds, in: Energy localisation and transfer, eds Dauxois T, Litvak-Hinenzon A, MacKay RS, Spanoudaki A (World Sci, 2004) 149-92.

[M05] MacKay RS, Indecomposable coupled map lattices with non-unique phase, in: Dynamics of coupled map lattices and of related spatially extended systems, eds Chazottes J-R, Fernandez B, Lect Notes Phys 671 (Springer, 2005) 65-94.

[M07a] MacKay RS, Parameter dependence of Markov processes on large networks, in: European Conference on Complex Systems 07 proceedings (2007), eds Jost J et al, article p41

[M07b] MacKay RS, Langevin equation for slow degrees of freedom of a Hamiltonian system, subm to proc. Mathematics of Model Reduction conference, Leicester, 2007

[MM] MacKay RS, MacKay DJC, Ergodic pumping: a mechanism to drive biomolecular conformation changes, Physica D 216 (2006) 220-34.

[MS] MacKay RS, Sepulchre J-A, Multistability in networks of weakly coupled bistable units, Physica D 82 (1995) 243-54.

[MSS] MacKay RS, Slijepcevic S, Stark J, Optimal scheduling in a periodic environment, Nonlinearity 13 (2000) 257-97.

[Man] Manson SM, Simplifying complexity: a review of complexity theory, Geoforum 32 (2001) 405-414.

[MK] Morita H, Kaneko K, Collective oscillation in a Hamiltonian dynamical system, Phys Rev Lett 96 (2006) 050602.

[PLOK] Politi A, Livi R, Oppo G-L, Kapral R, Unpredictable behavior of stable systems, Europhys Lett 22 (1993) 571-6.

[Ro] Roughgarden T, Selfish routing and the price of anarchy (MIT, 2005).

[RSV] Rybko A, Shlosman S, Vladimirov A, Spontaneous resonances and the coherent states of the queuing networks, arXiv:0708.3073v1[math-ph] (2007). 
[S+] Strogatz SH, Abrams DM, McRobie A, Eckhardt B, Ott E, Crowd synchrony on the millenium bridge, Nature 438 (2005) 43-4.

[Su] Sumpter DJT, The principles of collective animal behaviour, Phil Trans Roy Soc Lond B 361 (2006) 5-22.

[To+] Toom AL et al, Discrete local Markov systems, in: Stochastic cellular systems, ergodicity, memory, morphogenesis, eds Dobrushin RL, Kryukov VI, Toom AL (Manchester, 1990).

[Ya] Yarotsky DA, Quasiparticles in weak perturbations of non-interacting quantum lattice systems, arXiv: math-ph/0411042. 